\title{
No surviving evolved companions to the progenitor of supernova SN 1006
}

Jonay I. González Hernández ${ }^{1,2}$, Pilar Ruiz-Lapuente ${ }^{3,4}$, Hugo M. Tabernero ${ }^{5}$, David Montes $^{5}$, Ramon Canal ${ }^{4}$, Javier Méndez ${ }^{4,6}$, Luigi R. Bedin ${ }^{7}$

${ }^{1}$ Instituto de Astrofísica de Canarias, E-38205 La Laguna, Tenerife, Spain

2 Departamento de Astrofísica, Universidad de La Laguna, E-38206 La Laguna, Tenerife, Spain

${ }^{3}$ Instituto de Física Fundamental, CSIC, E-28006 Madrid, Spain

${ }^{4}$ Department of Astronomy, Institut de Ciències del Cosmos, Universitat de Barcelona (UB-IEEC), Martí i Franquès 1, E-08028 Barcelona, Spain

${ }^{5}$ Departamento de Astrofísica y Ciencias de la Atmósfera, Facultad de Ciencias Físicas, Universidad Complutense de Madrid, E-28040 Madrid, Spain

${ }^{6}$ Isaac Newton Group of Telescopes, P.O. Box 321; E-38700 Santa Cruz de La Palma, Spain.

7 INAF-Osservatorio Astronomico di Padova, Vicolo dell'Osservatorio 5, I-35122 Padova, Italy

Type Ia supernovae are thought to occur as a white dwarf made of car- 
bon and oxygen accretes sufficient mass to trigger a thermonuclear explosion ${ }^{1}$. The accretion could occur slowly from an unevolved (main-sequence) or evolved (subgiant or giant) $\operatorname{star}^{2,3}$, that being dubbed the single-degenerate channel, or rapidly as it breaks up a smaller orbiting white dwarf (the double- degenerate channel $)^{3,4}$. Obviously, a companion will survive the explosion only in the single-degenerate channel ${ }^{5}$. Both channels might contribute to the production of type Ia supernovae ${ }^{6,7}$ but their relative proportions still remain a fundamental puzzle in astronomy. Previous searches for remnant companions have revealed one possible case for SN $1572^{8,9}$, though that has been criticized $^{10}$. More recently, observations have restricted surviving companions to be small, main-sequence stars ${ }^{11,12,13}$, ruling out giant companions, though still allowing the single-degenerate channel. Here we report the result of a search for surviving companions to the progenitor of SN $1006^{14}$. None of the stars within 4 , of the apparent site of the explosion is associated with the supernova remnant, so we can firmly exclude all giant and subgiant companions to the progenitor. Combined with the previous results, less than 20 per cent of type Iae occur through the single degenerate channel.

Together with SN 1572 (Tycho Brahe's supernova), SN 1604 (Kepler's supernova) and the recently identified SN 185, SN 1006 is one of only four known historical Galactic type Ia supernova events (SNeIa). It is also the only one whose Ia type has never been disputed. While a survey of the stars close to the centre of Tycho's supernova remnant (SNR) produced a likely candidate for the SN companion ${ }^{8,9}$ and thus may be attributed 
to the single-degenerate channel, the absence of any ex-companion in the supernova remnant SNR 0509-67.5, in the Large Magellanic Cloud (LMC), down to very faint magnitudes, strongly suggests that the SN explosion there was produced by a double-degenerate system $^{15}$. Although the aforementioned direct searches have excluded, up to now, redgiant companions, there is some evidence from nearby spiral galaxies that a fraction of SNeIa may have had companions of this type ${ }^{16}$. This hypothesis, however, is challenged by the absence of ultraviolet emission that would be expected at the beginning of the SN outburst $^{17}$.

The distance to the remnant of SN $1006(2.18 \pm 0.08 \mathrm{kpc}$ away, as determined from the expansion velocity and the proper motion of the ejecta $\left.{ }^{18}\right)$, is much more precisely known than that of SN 1572 SNR $(2.83 \pm 0.70 \mathrm{kpc})$. The interstellar extinction is also much smaller, which makes the distances to the stars less uncertain. To be a possible candidate, a star must first be at the correct distance and, depending on its spectral type and luminosity class, show some spectral peculiarity, or an enhancement of the abundances of Fe-peak elements such as that seen in star G of Tycho ${ }^{9}$. Because of its relative vicinity compared with SN 1572 and the lower extinction $\left(A_{v}=0.3 \mathrm{mag}\right.$, vs. 2.0-2.4 mag in Tycho), going down to a red magnitude $m_{R}=15$ allows us to include, up to the SN distance and beyond, all red giants, all subgiants, and also main-sequence stars down to an absolute magnitude $M_{R} \simeq+3$.1. Inspecting the $2 \mathrm{MASS}^{19}$ photometric catalogue for possible unevolved companions (Supplementary Information), we found no main-sequence stars brighter that $m_{R} \simeq 16.4$, which brings the limit down to $M_{R} \simeq+4.5$, corresponding to $M_{V} \simeq+4.9$ (approximately equal to, or slightly less than, solar luminosity). Slightly 
evolved companions could be somewhat brigher: $m_{R} \simeq 16.0$ (just about twice as luminous as the Sun). Only in the case of SNR 0509-67.5 in the $\mathrm{LMC}^{15}$ has a fainter limit has been reached in a direct search.

We derive the stellar atmosphere parameters of the sample stars (Fig. 1) using the high-resolution UVES spectroscopic data (Table 1). The spectra of four giants, and F- and G-type dwarfs of the sample are shown in Fig. 2, and in Supplementary Fig. S2, respectively. In Table 1 we also provide the radial velocities of sample stars measured from the UVES spectra, and distances determined from five photometric magnitudes, taking into account the stellar parameters (Supplementary Information). In Fig. 3 we compare the abundances of Fe-peak elements in the stars of our sample with the Galactic $\operatorname{trends}^{26}$, for F-, G- and K-type unevolved stars. Unlike in SN 1572, where star G shows an overabundance of $\mathrm{Ni},[\mathrm{Ni} / \mathrm{Fe}]=0.16 \pm 0.04\left(\right.$ Here $[\mathrm{A} / \mathrm{B}]=\log \left(N_{A} / N_{B}\right)-\log \left(N_{A} / N_{B}\right) \odot$ for the number $N$ of atoms of elements $\mathrm{A}$ and $\mathrm{B}$, and subscript $\odot$ indicates the solar value), the stars in SN 1006 are all within the dispersion of the [Ni/Fe] Galactic trend. No enhancement is seen for any other element. In Supplementary Fig. S3, we also show the Galactic trends of several $\alpha$-elements and we do not see any clear anomaly in these element abundances either.

None of the stars in the sample shows any significant rotation. High rotation speeds have been claimed to be a characteristic of the surviving companions of $\mathrm{SNeIa}^{10}$, based on the assumptions that, owing to tidal interaction and in spite of the angular mometum loss due to mass transfer, the rotation periods before explosion are equal to the (short) orbital periods, and that the radius of the star remains basically unchanged after 
the explosion ${ }^{9}$. It has recently been shown ${ }^{27}$, however, that the impact of the SNIa ejecta on the companion does indeed reduce those speeds by a large factor, which would make the rotation criterion irrelevant.

Only four stars are at distances (marginally) compatible with that of the SNR. All of them are red giants: B16564 (G9-K0 III), B97341 (G9 III), B99810 (K1 III) and B93571 (K1 III) and none shows any spectroscopic peculiarity.

Two-dimensional hydrodynamic simulations of the impact of the ejecta of a SNIa on a red-giant binary companion have been performed ${ }^{28}$. More recently, the emission of supernova debris, arising from their impact with a similar companion, has been also considered $^{17}$. As for the effects of the explosion on the companion star, the results agree: most of the envelope is stripped away and what is left are the degenerate core and a very small fraction (amounting to a few per cent) of the original envelope. This hydrogenrich envelope initially extends up to $\sim 350 \mathrm{R}_{\odot}$ and then contracts on a thermal timescale. The star should be evolving at constant luminosity $\left(\sim 10^{3} \mathrm{~L}_{\odot}\right)$ towards increasing effective temperatures for $10^{5}-10^{6} \mathrm{yr}$. The same conclusion is reached when extrapolating to the red-giant case the results of simulations of the impact of SNIa ejecta on a main-sequence companion $^{29}$ : the red giant should have been stripped off most of its hydrogen envelope. Nothing similar to any of the above four normal red giants would be seen. On the other hand, the peculiar type of objects that would result from the interaction of the SNIa ejecta with a red-giant companion would be luminous enough to have been seen at the distance of SN 1006 and within our magnitude limit. A subgiant star similar to star G in Tycho would equally have been seen, but the only three subgiants in the sample 
(B20292, B15360 and B14707) lie much closer than the SNR. We are thus left only with main-sequence stars, which lie at shorter distances than the SNR, which have luminosities similar to, or lower than, the solar luminosity, and which are not predicted by any of the hydrodynamic simulations of the impact of SNIa ejecta with either a main-sequence star or a subgiant. In these hydrodynamic simulations ${ }^{28}$, a main-sequence companion of 1 solar mass is puffed up and heated, reaching $\sim 5,000$ times the solar luminosity; it is then

predicted to contract and cool down on a thermal time scale. It has been found ${ }^{30}$ that the return to luminosities of the order of those prior to the explosion could be faster because of the short cooling times of the outermost layers of the star, but, even so, in only $\sim 1000$ yr the object does not have enough time to become dimmer than the Sun (Supplementary Information).

Accordingly, SN 1006, the brightest event ever observed in our Galaxy, should have been produced either by mass accretion from an unevolved star, similar to, or less massive than, the Sun (with the above caveats), or by merging with another white dwarf. Adding this result to the evidence from the other direct searches, the single-degenerate channel appears either to encompass only a clear minority of cases (20\% or less), or preferentially it involves main-sequence companions with masses more probably below that of the Sun.

\section{References}

1 Nomoto, K., Saio, H., Kato, M., \& Hachisu, I. Thermal Stability of White Dwarfs Accreting Hydrogen-rich Matter and Progenitors of Type Ia Supernovae. Astrophys. J., 663, 1269-1276 (2007) 
2 Patat, F., Chandra, P., Chevalier, R., et al. Detection of Circumstellar Material in a Normal Type Ia Supernova. Science, 317, 924-926 (2007)

3 Branch, D., Livio, M., Yungelson, L.R., Boffi, F., Baron, E. In search of the progenitors of Type Ia supernovae. Publ. Astron. Soc. Pacif., 107, 1019-1029 (1995)

4 Pakmor, R., Kromer, M., Röpke, F. K., et al. Sub-luminous type Ia supernovae from the mergers of equal-mass white dwarfs with mass 0.9 Msolar. Nature, 463, 61-64 (2010)

5 Ruiz-Lapuente, P. The quest for a supernova companion. Science, 276, 1813-1814 (1997)

6 Greggio, L. The rates of Type Ia supernovae - II. Diversity of events at low and high redshifts. Month. Not. Royal Astron. Soc., 406, 22-42 (2010)

7 Brandt, T.D., et al. The ages of type Ia supernova progenitors. Astrophys. J., 140, $804-816(2010)$

8 Ruiz-Lapuente, P. et al. The binary progenitor of Tycho Brahe's 1572 supernova. Nature, 431, 1069-1072 (2004)

9 González Hernández, J.I., et al. The chemical abundances of Tycho G in supernova remnant 1572. Astrophys. J., 691, 1-15 (2009)

10 Kerzendorf, W. et al. Subaru high resolution spectroscopy of star G in the Tycho supernova remnant. Astrophys. J, 701, 1665-1672 (2009) 
11 Nugent, P.E., et al. Supernova SN 2011fe from an exploding carbon-oxygen white dwarf star. Nature, 480, 344-347 (2011)

$12 \mathrm{Li}, \mathrm{W}$., et al. Exclusion of a luminous red giant as a companion star to the progenitor of supernova SN 2011fe. Nature, 480, 348-350 (2011)

13 Edwards, Z.I., Pagnotta, A., Schaefer, B.E. The progenitor of the type Ia supernova that created SNR 0519-69.0 in the Large Magellanic Cloud. Astrophys. J., 747, L19-L23 (2012)

14 Stephenson, F.R. SN 1006: the brightest supernova. Astron. Geophys, 51, 5.27-5.32 (2010)

15 Schaefer, B.E., Pagnotta, A. An absence of ex-companion stars in the type Ia supernova remnant SNR 0509-67.5. Nature, 481, 164-166 (2012)

16 Sternberg, A., et al. Circumstellar material in type Ia supernovae via sodium absorption features. Science, 333, 856-859 (2011)

17 Kasen, D. Seeing the collision of a supernova with its companion star. Astrophys. J., 708, 1025-1031 (2010)

18 Winkler, P.F., Gupta, G., Long, K.S. The SN 1006 remnant: optical proper motions, deep imaging, distance, and brightness at maximum. Astrophys. J., 585, 324-335 (2003)

19 Cutri, R. M., Skrutskie, M. F., van Dyk, S., et al. 2MASS All-Sky Catalog of Point Sources. VizieR Online Data Catalog, 2246, 0 (2003) 
20 Allen, G.E., Petre, R., Gotthelf, E.V. X-ray synchrotron emission from 10-100 TeV cosmic ray electrons in the supernova remnant SN 1006. Astrophys. J., bf 558, 739-752 (2001)

21 Monet, D.G. The 526,289,881 objects in the USNO-A2.0 Catalogue. Bull. Amer. Astron. Soc., 30, 1427 (1998)

22 Winkler, P.F., Long, K.S., Hamilton, A.J.S., Fesen, R.A. Probing multiple sight lines through the SN 1006 remnant by ultraviolet absorption spectroscopy. Astrophys. J., 624, 189-197 (2005)

23 Tabernero, H. M., Montes, D., González Hernández, J. I. Chemically tagging the Hyades Supercluster. A homogeneous sample of F6-K4 kinematically-selected northern stars. Astron. Astrophys., in press (2012). Preprint at (http://arxiv.org/abs/astro$\mathrm{ph} / 1245.4879)$

24 Sneden, C. PhD thesis. Univ. Texas, Austin (1973)

25 Kurucz, R.L. ATLAS89 Stellar Atmospheres Programs and $2 \mathrm{~km} \mathrm{~s}^{-1}$ Grid. (CDROM, Smithsonian Astrophysical Observatory, Cambridge) (1993)

26 Neves, V., Santos, N.C., Sousa, S.G., Correia, A.C.M, Israelian, G. Chemical abundances of 451 stars from the HARPS GTO planet search program. Thin disk, thick disk, and planets Astron. Astrophys., 497, 563-81 (2009)

27 Pan, K.-C., Ricker, P., Taam, R. Impact of type Ia supernova ejecta on the binary companions in the single-degenerate scenario. Astrophys. J., 750, 151 (2012) 
28 Marietta, E., Burrows, A., Fryxell, B. Type Ia supernova explosions in binary systems: the impact on the secondary star and its consequences. Astroph. J. Suppl., 128, 615-650 (2000)

29 Pakmor, R., Röpke, F.K., Weiss, A., Hillebrandt, W. The impact of Type Ia supernovae on main sequence binary companions. Astron. Astrophys., 489, 943-951 (2008)

30 Podsiadlowski, P. On the evolution and appearance of a surviving companion after a Type Ia supernova explosion. Preprint at (http://arxiv.org/abs/astro-ph/0303660) $(2003)$ 
Supplementary Information is linked to the online version of the paper at www.nature.com/nature.

Acknowledgements This work was supported by the Spanish Ministerio de Ciencia e Innovación (MICINN), the Universidad Complutense de Madrid (UCM), and the Comunidad de Madrid. This work is based on observations collected with the UVES spectrograph at the VLT/UT2 8.2-m Kueyen Telescope (ESO run ID. 69.D-0397(A)) at the Paranal Observatory, Chile. We are grateful to the Cerro Paranal Observatory staff and to the User Support Department of ESO for their help.

Author Contributions J.I.G.H. performed the chemical abundance analysis of the observed UVES spectra and determined the distances to the targets. J.I.G.H. and P.R.L. wrote the paper. P.R.L. was the Principal Investigator of the ESO proposal. R.C. and J.M. also participated in the ESO proposal. H.M.T. and D.M. derived the stellar parameters and created figures with the observed spectra. R.C. and P.R.L. contributed to the astrophysical interpretation of the results. J.M. and L.R.B. collected the photometric data and created figures with the field and the supernova remnant. All the authors provided helpful comments and contributed to improve the text of the final version paper.

Author information Correspondence and requests should be addressed to J.I.G.H and P.R.L (jonay@iac.es; pilar@am.ub.es) 
Table 1 | Parameters of the sample stars.

\begin{tabular}{llccrrc}
\hline \hline Name & $T_{\text {eff }}$ & $\log g$ & $v_{\text {turb }}$ & {$[\mathrm{Fe} / \mathrm{H}]$} & $v_{\text {rad }}$ & $d$ \\
\hline B09472 & $5 \mathrm{~K}]$ & {$[\mathrm{cgs}]$} & {$[\mathrm{km} / \mathrm{s}]$} & {$[\mathrm{dex}]$} & {$[\mathrm{km} / \mathrm{s}]$} & {$[\mathrm{kpc}]$} \\
B11408 & $4677 \pm 68$ & $4.52 \pm 0.17$ & $1.211 \pm 0.105$ & $-0.04 \pm 0.06$ & $-20.74 \pm 2.14$ & $1.23 \pm 0.53$ \\
B05723 & $5910 \pm 87$ & $4.50 \pm 0.23$ & $1.249 \pm 0.107$ & $0.30 \pm 0.07$ & $-12.81 \pm 3.06$ & $0.76 \pm 0.32$ \\
B17720 & $5300 \pm 86$ & $4.65 \pm 0.22$ & $1.190 \pm 0.193$ & $0.33 \pm 0.06$ & $-60.13 \pm 1.30$ & $0.58 \pm 0.25$ \\
B16564 & $4845 \pm 39$ & $3.13 \pm 0.16$ & $1.607 \pm 0.049$ & $-0.37 \pm 0.03$ & $-116.07 \pm 1.86$ & $3.03 \pm 1.27$ \\
B97338 & $5707 \pm 42$ & $4.21 \pm 0.12$ & $1.292 \pm 0.054$ & $0.01 \pm 0.04$ & $-5.53 \pm 2.25$ & $1.05 \pm 0.45$ \\
B05518 & $6327 \pm 63$ & $4.85 \pm 0.13$ & $1.386 \pm 0.090$ & $0.14 \pm 0.04$ & $-4.38 \pm 1.92$ & $0.50 \pm 0.21$ \\
B20292 & $5196 \pm 33$ & $3.67 \pm 0.09$ & $1.372 \pm 0.051$ & $-0.66 \pm 0.03$ & $2.92 \pm 2.52$ & $1.13 \pm 0.48$ \\
B14130 & $6177 \pm 44$ & $4.61 \pm 0.09$ & $1.528 \pm 0.052$ & $0.31 \pm 0.03$ & $-21.26 \pm 2.09$ & $0.89 \pm 0.38$ \\
B03395 & $5517 \pm 49$ & $4.36 \pm 0.13$ & $1.165 \pm 0.074$ & $0.22 \pm 0.04$ & $-56.18 \pm 5.21$ & $0.95 \pm 0.41$ \\
B97341 & $4881 \pm 51$ & $2.98 \pm 0.18$ & $1.641 \pm 0.057$ & $-0.16 \pm 0.04$ & $-41.76 \pm 1.96$ & $2.48 \pm 1.04$ \\
B99810 & $4658 \pm 39$ & $2.51 \pm 0.15$ & $1.782 \pm 0.039$ & $-0.72 \pm 0.03$ & $33.53 \pm 1.50$ & $2.42 \pm 1.02$ \\
B15360 & $4960 \pm 70$ & $3.40 \pm 0.21$ & $1.886 \pm 0.086$ & $0.07 \pm 0.05$ & $-37.03 \pm 1.61$ & $1.29 \pm 0.54$ \\
B93571 & $4579 \pm 58$ & $2.51 \pm 0.22$ & $2.182 \pm 0.066$ & $-0.21 \pm 0.05$ & $-100.33 \pm 1.58$ & $2.39 \pm 1.01$ \\
B18024 & $6083 \pm 58$ & $4.44 \pm 0.13$ & $1.545 \pm 0.095$ & $-0.36 \pm 0.04$ & $22.61 \pm 2.35$ & $0.60 \pm 0.26$ \\
B98824* & & & & & & \\
B08277 & $5693 \pm 34$ & $4.39 \pm 0.09$ & $1.123 \pm 0.044$ & $0.17 \pm 0.03$ & $-49.50 \pm 2.08$ & $0.45 \pm 0.19$ \\
B24215 & $5729 \pm 45$ & $4.44 \pm 0.12$ & $1.189 \pm 0.058$ & $0.16 \pm 0.04$ & $3.02 \pm 2.39$ & $0.95 \pm 0.41$ \\
B14707 & $5065 \pm 47$ & $3.36 \pm 0.15$ & $1.270 \pm 0.060$ & $0.16 \pm 0.04$ & $22.14 \pm 1.83$ & $1.37 \pm 0.58$ \\
B90474 & $5051 \pm 38$ & $3.05 \pm 0.12$ & $1.874 \pm 0.046$ & $-0.29 \pm 0.03$ & $-98.10 \pm 1.95$ & $4.78 \pm 2.00$ \\
B90102 & $5650 \pm 33$ & $4.21 \pm 0.10$ & $1.076 \pm 0.049$ & $-0.11 \pm 0.03$ & $85.57 \pm 1.16$ & $0.91 \pm 0.39$ \\
B10074 & $5601 \pm 63$ & $4.52 \pm 0.14$ & $1.036 \pm 0.102$ & $0.05 \pm 0.05$ & $-30.26 \pm 1.38$ & $0.71 \pm 0.30$ \\
B95979 & $6776 \pm 94$ & $4.19 \pm 0.16$ & $1.832 \pm 0.131$ & $0.02 \pm 0.06$ & $-32.94 \pm 6.81$ & $0.94 \pm 0.40$ \\
B09749 & $6163 \pm 65$ & $4.53 \pm 0.14$ & $1.362 \pm 0.082$ & $0.14 \pm 0.05$ & $-13.15 \pm 2.60$ & $0.40 \pm 0.17$ \\
B21185* & & & & & & \\
B26090 & $6101 \pm 39$ & $4.58 \pm 0.09$ & $1.238 \pm 0.053$ & $0.15 \pm 0.03$ & $-48.08 \pm 2.52$ & $0.37 \pm 0.16$ \\
\hline & & & & & & \\
\hline
\end{tabular}

*Spectroscopic binary 


\section{TABLE CAPTIONS}

Table 1 | Parameters of the sample stars. We provide the stellar atmosphere parameters (effective temperature, $T_{\text {eff }}$, surface gravity, $\log g$, and microturbulent velocity,

$\left.v_{\text {turb }}\right)$, metallicities, $[\mathrm{Fe} / \mathrm{H}]$, radial velocities, $v_{\text {rad }}$, and distances, $d$, of the sample stars, together with 1- $\sigma$ uncertainties. The stellar parameters and metallicities were obtained from Fe I and Fe II excitation and ionization equilibria, using the STEPAR ${ }^{23}$ code, which makes use of the MOOG $^{24}$ code and ATLAS9 model atmospheres ${ }^{25}$. Our analysis has been performed assuming local thermodynamic equilibrium (LTE). SN 1006 is located about 500 pc above the Galactic plane. Unlike the case of SN 1572, which lies close to the Galactic plane, the radial velocities of the stars along the line of sight do not follow a regular pattern (this can be seen in the sixth column), since most of them belong either to the thick disc or to the halo of the Galaxy (see the dispersion in metallicities in the fifth column). 


\section{FIGURE CAPTIONS}

Figure 1 | DSS2 R-band image of the SN 1006 field. The positions and names of the 26 stars included in the spectroscopic survey (selected from the USNO-A2.0 Catalogue ${ }^{21}$ ) are given. The centre of our search is the geometrical centre of the quite symmetrical X-ray emission of the $\mathrm{SNR}^{20}$ (green cross), at $\mathrm{RA}=15^{h} 2^{m} 55^{s}$, Dec $=-41^{\circ} 55^{\prime}$ $12^{\prime \prime}$. Also shown are the boundary of the surveyed region (large green circle) and the geometrical centre of the H-alpha emission ${ }^{18}$ (small yellow circle). Giant stars are marked by red squares. See also Supplementary Fig. S1, for a full view of the SNR. Another centre has been proposed more recently ${ }^{22}$, based on the distribution of the ejecta along the line of sight. It is, however, still located within our surveyed area. For a star at a distance $d \simeq 2.2$ $\mathrm{kpc}$ and a velocity perpendicular to the line of sight of $\sim 100 \mathrm{~km} \mathrm{~s}^{-1}$ (roughly the orbital velocity before the explosion), the angular displacement on the sky in 1000 years would be $10^{\prime \prime}$ only. However, given the asymmetry of the SNR, and also that in core-collapse SNe the distance between the compact object and the X-ray centroid of the SNR can be $15 \%$ of the radius of the SNR or more, we adopted a much wider radius for the search: $4^{\prime}$. That amounts to $27 \%$ of the radius of the SNR, which is $15^{\prime}$ (Supplementary Fig. S1). Positions, magnitudes and angular distances to the centre of the survey are given in Supplementary Table S1.

Figure 2| Observed UVES spectra of the candidate giant stars. The spectra are labelled by their names in Table 1 and sorted in decreasing effective temperature order from top to bottom. These high-resolution spectra were obtained at the 8.2-m Kueyen 
VLT (UT2) telescope equipped with UVES at the Cerro Paranal Observatory in Chile. They were obtained on 13, 14, 25, 28-30 April 2002 and on 1 May 2002, covering the

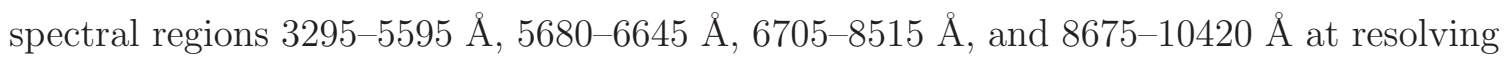
power $\lambda / \Delta \lambda \approx 43000$. The $\mathrm{S} / \mathrm{N}$ of the spectra is in the range $50-170$ and typically 80 . The data were reduced in a standard manner, and later normalized within IRAF, using low-order polynomial fits to the observed continuum.

Figure 3 | Stellar abundance ratios $[\mathrm{X} / \mathrm{Fe}]$ of several Fe-peak elements. The chemical abundances have been derived using the EW technique for all of the elements. We performed a differential analysis on a line-by-line basis, using the solar UVES spectrum of the Moon as reference (see the Supplementary Section 3 for more details). The abundances of several Fe-peak elements are listed in Supplementary Table S4b relative to iron are compared with the Galactic trends of these elements in the relevant range of metallicities ${ }^{26}$. Red triangles correspond to the four giant stars whose distances are marginally compatible with that of the remnant of SN 1006. Blue squares, to the rest of the stars in the sample. The error bars are the 1- $\sigma$ uncertainties associated with the dispersion of the measurements from different spectral features. 


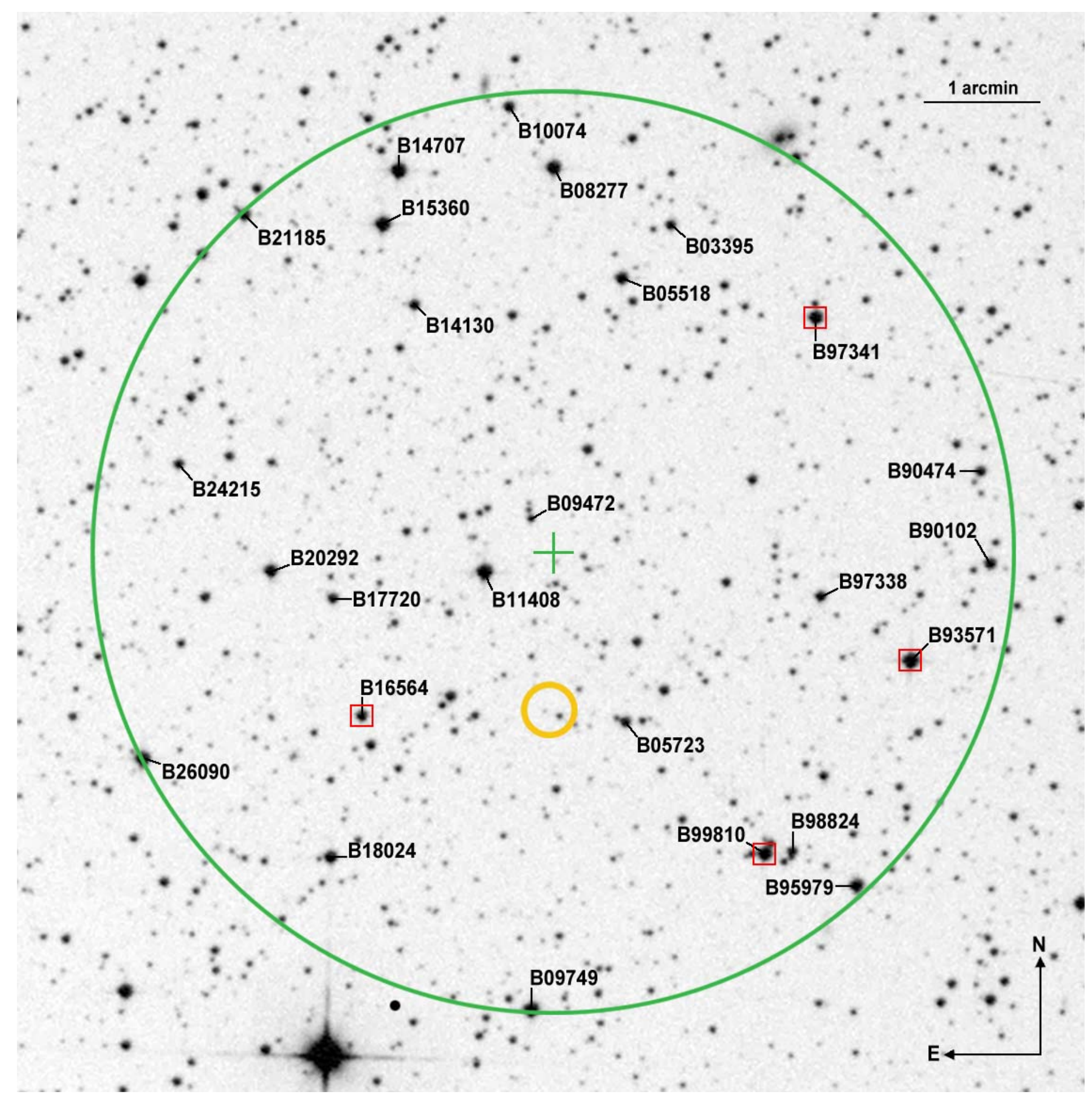




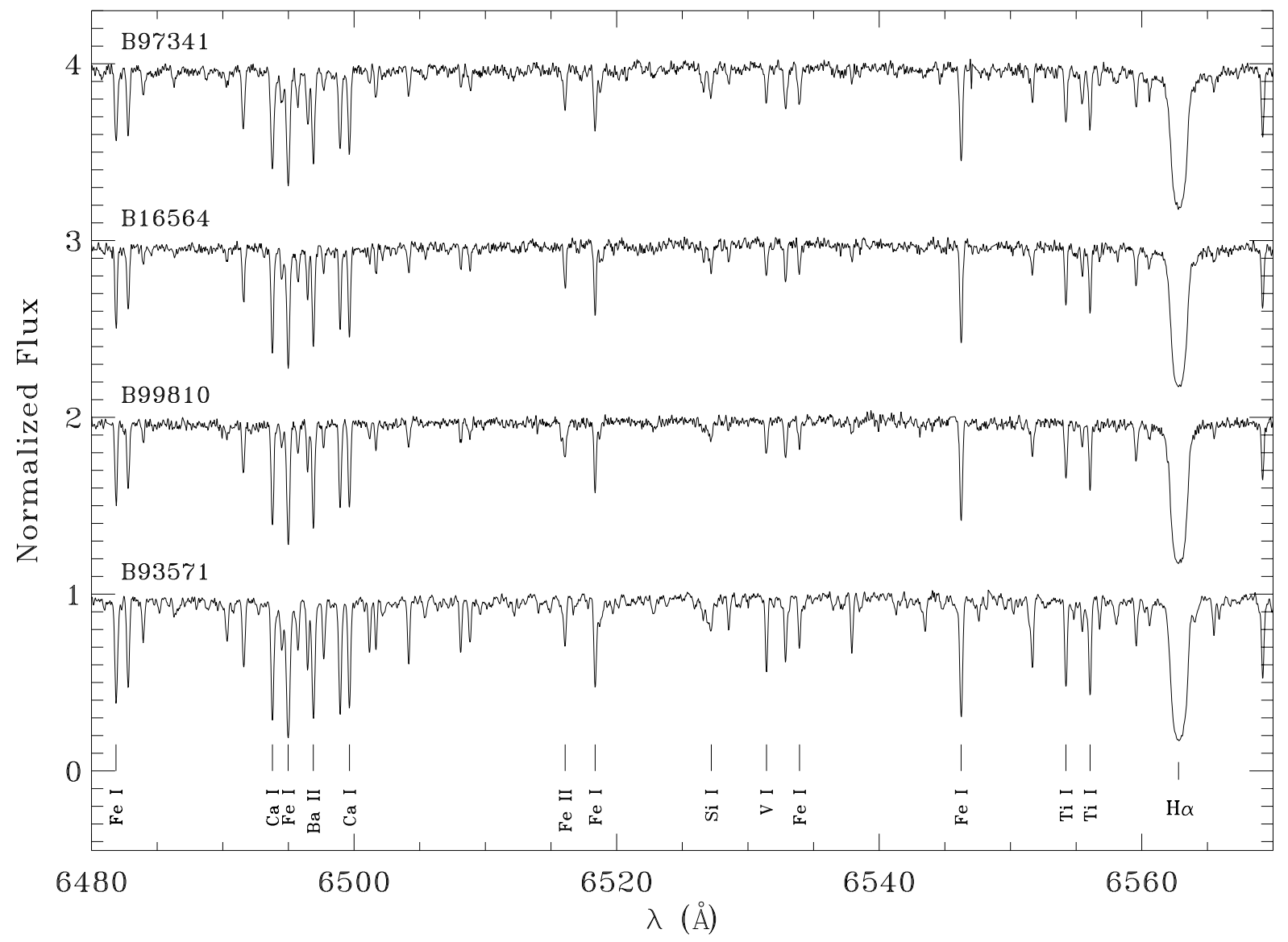



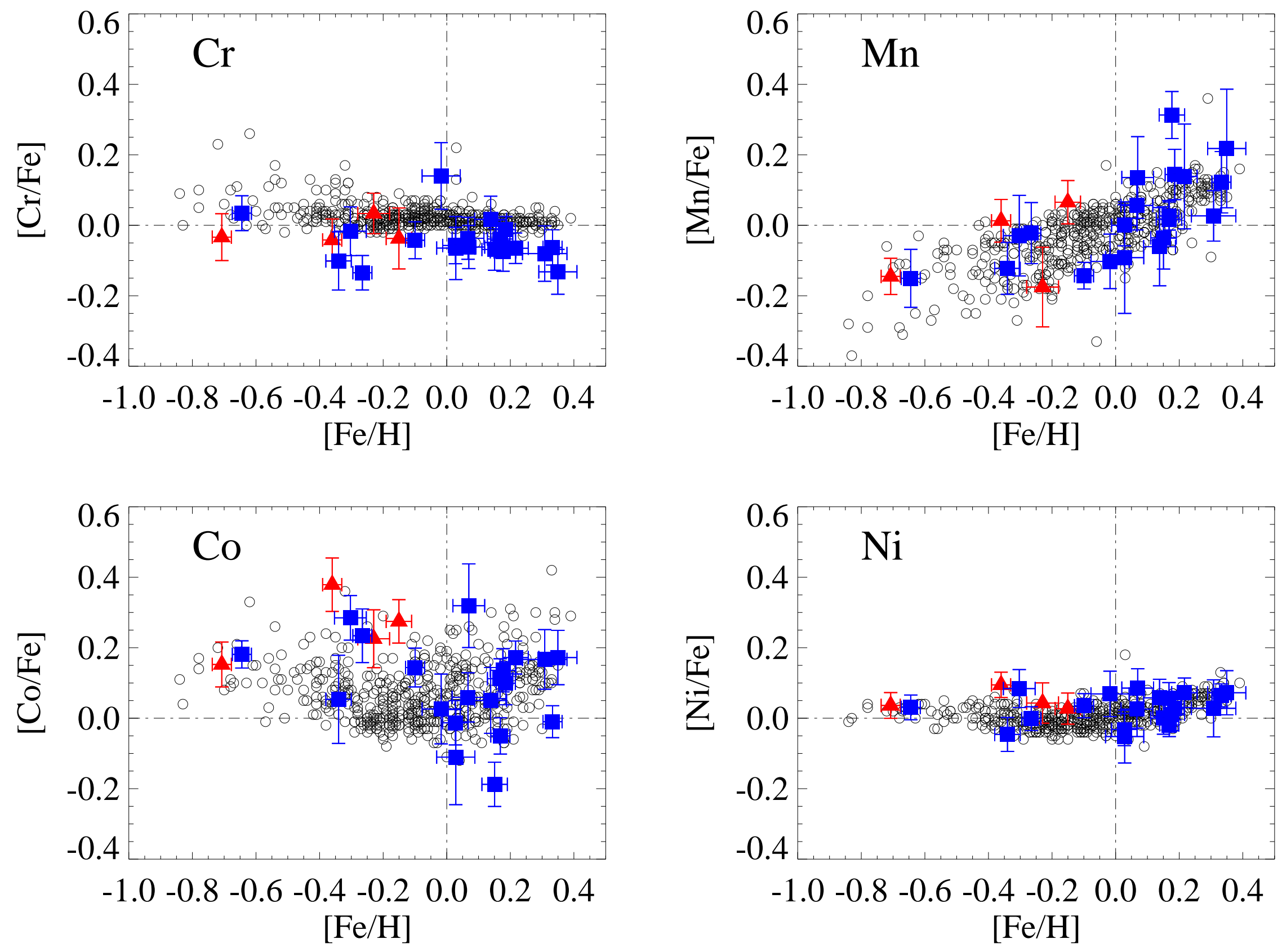